\title{
Geometric lattice actions, entropy and fundamental groups
}

\author{
David Fisher ${ }^{1}$ and Robert J. Zimmer ${ }^{2}$
}

\begin{abstract}
Let $\Gamma$ be a lattice in a noncompact simple Lie Group $G$, where $\mathbb{R}-\operatorname{rank}(G) \geq 2$. Suppose $\Gamma$ acts analytically and ergodically on a compact manifold $M$ preserving a unimodular rigid geometric structure (e.g. a connection and a volume). We show that either the $\Gamma$ action is isometric or there exists a "large image" linear representation $\sigma$ of $\pi_{1}(M)$. Under an additional assumption on the dynamics of the action, we associate to $\sigma$ a virtual arithmetic quotient of full entropy.
\end{abstract}

Mathematics Subject Classification (2000). 57S20, 53C24, 22F50.

Keywords. Lattices in Lie groups, rigidity, rigid geometric structures, entropy.

\section{Introduction}

Suppose $G$ is a noncompact simple Lie group and $G$ acts analytically on a compact manifold $M$ preserving a unimodular rigid geometric structure, for example either a connection and a volume form or a pseudo-Riemannian metric. Assuming the action is ergodic, Gromov [G] constructed a linear representation $\sigma: \pi_{1}(M) \rightarrow G L_{n}(\mathbb{R})$ such that the Zariski closure of $\sigma\left(\pi_{1}(M)\right)$ contains a group locally isomorphic to $G$. One cannot hope for an analogous result for lattices in all semi-simple groups, since lattices in rank 1 groups often admit homomorphisms to $\mathbb{Z}$, and many counterexamples can be constructed for $\mathbb{Z}$ actions. In addition, even for lattices in higher rank simple groups, there exist isometric actions on manifolds with finite fundamental group. Here we prove a form of Gromov's theorem for lattices, which shows that for actions of higher rank lattices either the action is isometric or there exists a representation like Gromov's. More precisely:

Theorem 1.1. Let $\Gamma<G$ be a lattice, where $G$ is a simple group and $\mathbb{R}-$ $\operatorname{rank}(G) \geq 2$. Suppose $\Gamma$ acts analytically and ergodically on a compact manifold $M$ preserving a unimodular rigid geometric structure. Then either

\footnotetext{
${ }^{1}$ Partially supported by NSF grant DMS-9902411.

${ }^{2}$ Partially supported by NSF grant.
} 
i) the action is isometric and $M=K / C$ where $K$ is a compact Lie group and the action is by right translation via $\rho: \Gamma \hookrightarrow K$, a dense image homomorphism, or

ii) there exists an infinite image linear representation $\sigma: \pi_{1}(M) \rightarrow G L_{n} \mathbb{R}$, such that the algebraic automorphism group of the Zariski closure of $\sigma\left(\pi_{1}(M)\right)$ contains a group locally isomorphic to $G$.

To prove the theorem, one first applies Gromov's result to the induced $G$ action on $(G \times M) / \Gamma$ to obtain a linear representation $\sigma$ of $\Lambda=\pi_{1}((G \times M) / \Gamma)$. However, $\pi_{1}(M)$ is a normal subgroup of $\Lambda$, and in fact, if $\pi_{1}(G)$ is trivial, we have a short exact sequence:

$$
1 \longrightarrow \pi_{1}(M) \longrightarrow \Lambda \longrightarrow \Gamma \longrightarrow 1
$$

To show that the restriction of $\sigma$ to $\pi_{1}(M)$ is trivial only when the $\Gamma$ action is isometric, we use the techniques of [Z5] to compare the entropy of the $G$ action on $(G \times M) / \Gamma$ with the image $\sigma(\Lambda)$. If $G$ is not simply connected, let $\pi: \tilde{G} \rightarrow G$ be the universal cover and $\tilde{\Gamma} \rightarrow \Gamma$ the pre-image of $\Gamma$ under the covering map $\pi$. The exact sequence above now holds with $\tilde{\Gamma}$ in place of $\Gamma$. By viewing the $\Gamma$ action as a $\tilde{\Gamma}$ action via the homomorphism $\tilde{\Gamma} \rightarrow \Gamma$, we can always assume that our lattice $\Gamma$ is a lattice in a simply connected group $G=\tilde{G}$. We will make this assumption throughout this paper.

There is one earlier result along these lines for lattice actions. In [Z3], the second author shows that, under the assumptions of Theorem 1.1, if we assume $\pi_{1}(M)$ is trivial, then the action is isometric. As a corollary, we obtain the following generalization of this fact:

Corollary 1.2. Let $\Gamma$ act on $M$ as in Theorem 1.1. Further assume there are no infinite image linear representation of $\pi_{1}(M)$. Then the $\Gamma$ action on $M$ is isometric and $\pi_{1}(M)$ is finite.

An ergodic action of $G$ on $M$ is said to be engaging if any lift of the action to a finite cover of $M$ is still ergodic. In [LZ], given an engaging action on $M$ of a simple group $G, \mathbb{R}-\operatorname{rank}(G) \geq 2$, the authors associate a virtual arithmetic quotient to any linear representation of $\pi_{1}(M)$. Using the explicit construction of Gromov's representation $\sigma$, the second author has shown that for $G$ actions which are analytic and preserve a rigid geometric structure, the associated virtual arithmetic quotient has the same entropy as the original $G$ action [Z5]. Using the results of $[\mathrm{F}]$, we prove the following analogous result here:

Theorem 1.3. Assume $\Gamma$ acts on $M$ as in Theorem 1.1. Further assume the action is engaging. Then either

i) The $\Gamma$ action is isometric and is described as in Theorem 1.1, or

ii) For some finite index subgroup $\Gamma^{\prime}<\Gamma$ and some finite cover, $M^{\prime}$ of $M$, there is a measurable $\Gamma^{\prime}$ equivariant map $\varphi: M^{\prime} \rightarrow K \backslash L / L_{\mathbb{Z}}$. If $h_{-}$is the entropy function of the relevant $\Gamma$ action, we have $h_{M}(\gamma)=h_{K \backslash L / L_{Z}}(\gamma)$ for all $\gamma \in \Gamma^{\prime}$. 
Here the $\Gamma^{\prime}$ action on $K \backslash L / L_{\mathbb{Z}}$ is a generalized affine action.

We recall that an affine diffeomorphism $f$ of a homogeneous space $A / B$ is simply one that is covered by a diffeomorphism $\tilde{f}$ of $A$, where $\tilde{f}$ is the composition of a group automorphism $L$ and left translation by an element of $A$. For this to make sense it is clear that $L(B)=B$. A group action on a homogeneous space is said to be affine if every element in the group acts by an affine diffeomorphism. The generalized affine action of Theorem 1.3. refers to the fact that we are not acting on a homogeneous space. Instead the action is on a quotient of the homogeneous space by a compact group of affine diffeomorphisms via diffeomorphisms covered by affine diffeomorphisms of the homogeneous space. More precisely:

Definition 1.4. Let $A / B$ be a homogeneous space and $D$ and $F$ two commuting groups of affine diffeomorphisms of $A / B$, with $D$ compact. The we call the $F$ action on $D \backslash A / B$ a generalized affine action.

The proof of Theorem 1.3. gives a more detailed description of the action. The action is shown to be arithmetic in the sense of Definition 3.2. of [F]. That any generalized affine action of a lattice in a higher rank simple group is arithmetic is also a straightforward exercise from Margulis' superrigidity theorem and the structure theory of algebraic groups.

Note that two frequently studied examples of generalized affine actions of higher rank lattices are actions by left translations on homogeneous spaces and affine actions on nilmanifolds. In the latter case the affine diffeomorphisms considered generally have no translational part (at least on a subgroup of finite index). In general, one can construct actions that fall into neither of these two categories, i.e. affine actions where group elements act by compositions of non-trivial translations and non-trivial automorphisms.

We now sketch the proof of Theorem 1.1. For ease of exposition, we assume that $\Lambda=\pi_{1}((G \times M) / \Gamma)=\Gamma \ltimes \pi_{1}(M)$, i.e. that the extension above is split. In order to apply Gromov's result to the $G$ action on $(G \times M) / \Gamma$ we need to produce a rigid geometric structure $\omega$ invariant under this action. This will be locally a product of the given structure on $M$ with the natural pseudo-Riemannian structure along the $G$ orbits given by the Killing form on $\mathfrak{g}$. In fact, there does not seem to be a definition in the literature of a product of geometric structures in the sense of Gromov or a proof that a product of rigid structures is rigid. In the case of Cartan's geometric structures of finite type this is classical, and we will give the analogous definitions and proofs for the more general setting in Section 3.

An important property of this particular geometric structure on $(G \times M) / \Gamma$ is that we can explicitly identify a Lie algebra $\mathfrak{g}$ of local Killing fields of the structure commuting with the $G$ action. These come from lifting the structure $\omega$ to a structure $\tilde{\omega}$ on the universal cover $G \times \tilde{M}$ and then differentiating the right $G$ action on $G$, which preserves the structure by construction. 
Gromov's representation $\sigma$ is constructed on all Killing fields $V$ of $\tilde{\omega}$ commuting with the lift of the $G$ action. Ideally, one would like to be able to say that $V$ splits as the sum of $\mathfrak{g}$ above and a collection of Killing fields $W$ tangent to the $M$ fibers in $(G \times M) / \Gamma$. This would immediately give a representation of $\pi_{1}(M)$ on $W$. Regrettably, it is not clear how to construct such Killing fields in a natural way that would allow one to show that this representation is non-trivial, and one must approach the problem indirectly. It follows from the structure of the $\pi_{1}((G \times M) / \Gamma)$ action on $G \times \tilde{M}$ (as described in [F]) that $\sigma\left(\pi_{1}(M)\right)$ acts trivially on the Lie algebra $\mathfrak{g}$ of Killing fields constructed above and that $\sigma(\Gamma)$ acts via the adjoint representation of $G$. What remains to be shown is that if $\sigma\left(\pi_{1}(M)\right)$ is not infinite, then the action is isometric.

To do this, we compare the entropies of various actions and representations. First, in Section 2, we observe that the entropy of the induced action is the sum of the entropy of the $\Gamma$ action on $M$ and the entropy of the $G$ action on $G / \Gamma$. (This is precisely true if we induce and then restrict to the $\Gamma$ action. A stronger statement will be made precise in Section 2.)

From this it follows that if the entropy of the induced action equals the entropy of the $G$ action on $G / \Gamma$, the $\Gamma$ action must have zero entropy. An adaptation of standard arguments then shows that actions preserving a rigid geometric structure and having zero entropy are isometric.

To complete the proof, we show that unless the $\sigma\left(\pi_{1}(M)\right)$ has large Zariski closure, the entropy of the induced action is indeed equal to the entropy of the $G$ action on $G / \Gamma$. This is done using an estimate from [Z5]. Gromov has shown that the Lie algebra, $\mathfrak{l}$, of the Zariski closure of $\sigma\left(\pi_{1}((G \times M) / \Gamma)\right)$ has a natural structure as a $G$ module. In [Z5], the second author shows that the entropy of this module is an upper bound on the entropy of the $G$ action. (The notion of entropy of a module is made precise in Section 4.) The Lie algebra of the Zariski closure of $\sigma\left(\pi_{1}(M)\right)$ is a $G$ submodule of $\mathfrak{l}$. If the entropy of this submodule is zero, then the entire entropy of $\mathfrak{l}$ comes from the Lie algebra of the Zariski closure of $\sigma(\Gamma)$. An explicit analysis of the construction of Gromov's representation shows that the entropy of this module is equal to the entropy of the $G$ action on $G / \Gamma$. This analysis uses the existence of our explicitly constructed Lie algebra $\mathfrak{g}$ of Killing fields along the $G$ orbits in $(G \times M) / \Gamma$. This shows that the Lie algebra of the Zariski closure of $\sigma\left(\pi_{1}(M)\right)$ is a non-trivial $G$ module unless the $\Gamma$ action is isometric, and we are done.

To prove Theorem 1.3 we use a similar analysis of Gromov's representation for induced actions to adapt the arguments of $[\mathrm{F}]$ and [Z5] to the present setting. We first show that the induced action has a full entropy quotient and then use techniques of $[\mathrm{F}]$ to show that this quotient has the structure of an induced action. The techniques of $[\mathrm{F}]$ produce an arithmetic quotient $Y$ of the $\Gamma$ action, and show that the quotient for the induced action on $(G \times M) / \Gamma$ is actually the induced action on $(G \times Y) / \Gamma$. Theorem 2.3 then tells us that the $\Gamma$ entropy on $Y$ must equal the $\Gamma$ entropy on $M$. 
The first author would like to thank E. Benveniste, R. Feres and G. Margulis for enlightening conversations regarding rigid geometric structures. Both authors thank the Newton Institute for Mathematical Sciences for it's hospitality during the time when this work was completed and the first author also thanks ETH Zurich.

\section{Computing entropy}

In this section, we discuss the computation of entropy for actions of lattices and the relation to the entropy of the induced action. The basic tool is the use of superrigidity to linearize the derivative cocycle. Let $P \rightarrow M$ be a principal $H$ bundle where $H$ is a connected real algebraic group. Assume a group $G$ acts on $P$ by automorphisms preserving a finite measure on $M$. Let $\sigma: G \rightarrow H$ be a homomorphism. We say that a measurable section $s: M \rightarrow P$ is $\sigma$-simple if there exists a cocycle $c: M \times G \rightarrow K$ where $K<Z_{H}(\sigma(G))$ is compact and

$$
s(g m)=g s(m) \sigma(g)^{-1} c(m, g)
$$

for all $g \in G$ and almost all $m \in M$. Let $G$ be a simple Lie group, $\mathbb{R}-\operatorname{rank}(G) \geq$ 2, let $M$ be a compact space and assume the $G$ action on $M$ is ergodic and continuous. Superrigidity for cocycles implies that there exists a finite ergodic extension $X \rightarrow M$, so that for the action on $P^{\prime}$, the pullback of $P$ to $X$, there exists a representation $\sigma: G \rightarrow H$ and a $\sigma$-simple section $s$. In fact, we need not assume that $M$ is compact but only that the cocycle defined by the $G$ action on $P$ is quasi-integrable, a boundedness condition that will hold in all situations of interest here (see [Z6] for details). We will refer to $\sigma$ as the superrigidity representation. If $\Gamma<G$ is a lattice and we have an action of $\Gamma$ on $P$ which is ergodic on $M$ (with no $G$ action) then we have a very similar conclusion. Either, for some finite ergodic extension there is a linear representation $\sigma: G \rightarrow H$ and a $\sigma$-simple section or the algebraic hull of the $\Gamma$ action on $P$ is compact. Here again we require either $M$ compact or the cocycle defined by the $\Gamma$ action quasi-integrable.

Applying superrigidity to the derivative action on the frame bundle, we can compute the entropy directly via Pesin's formula as in the following two theorems from $[\mathrm{Z} 1]$ :

Theorem 2.1. Suppose $G$ is a simple Lie group with $\mathbb{R}-\operatorname{rank}(G) \geq 2$. Suppose $G$ acts ergodically on a manifold $M$ by diffeomorphisms preserving a $C^{2}$ volume form such that the volume of $M$ is finite. Further assume that the derivative cocycle is quasi-integrable. For each $g \in G$, let $h(g)$ be the entropy. Let $\sigma: G \rightarrow S L_{n} \mathbb{R}$ be the superrigidity representation for the action on the frame bundle of $M, n=\operatorname{dim} M$. Then $h(g)=\Sigma \log ^{+}\|\lambda\|$, where $\lambda$ runs over all eigenvalues of $\sigma(g)$.

Theorem 2.2. Suppose $\Gamma<G$ is a lattice, $G$ as above. Suppose $\Gamma$ acts ergodically by diffeomorphisms on a compact manifold $M$ preserving a $C^{2}$ volume form. For 
each $\gamma \in \Gamma$ let $h(\gamma)$ be the entropy. Let $\sigma: G \rightarrow S L_{n} \mathbb{R}$ be the superrigidity representation for the action on the frame bundle of $M, n=\operatorname{dim} M$. Then $h(\gamma)=$ $\Sigma \log ^{+}\|\lambda\|$, where $\lambda$ runs over all eigenvalues of $\sigma(\gamma)$, or the algebraic hull of the derivative cocycle is compact and $h(\gamma)=0$ for all $\gamma \in \Gamma$.

The proof of this is essentially in [Z1], though there $M$ is always assumed to be compact in Theorem 2.1. Compactness is used to be able to apply Pesin's formula which is often only stated for actions on compact manifolds, but which holds whenever the action preserves a finite volume and the derivative cocycle is quasi-integrable [P1, P2]. We apply Theorem 2.1 to the derivative cocycle of the $G$ action on $(G \times M) / \Gamma$. Though $(G \times M) / \Gamma$ is not compact when $\Gamma$ is not cocompact, the derivative cocycle is still quasi-integrable by the results in $[\mathrm{L}]$ if $G$ is algebraic and by results in Section 7 of $[\mathrm{F}]$ if $G$ is not algebraic.

Suppose $\Gamma$ acts on $M$ by $C^{2}$ diffeomorphisms preserving volume. The induced $G$ action on $(G \times M) / \Gamma$ is also $C^{2}$ and volume preserving, and the $G$ action clearly preserves the decomposition $T((G \times M) / \Gamma)=T(\mathfrak{G}) \times T M$ where $T(\mathfrak{G})$ is the tangent space to the $G$ orbits and the direct product is given by the fact that $(G \times M) / \Gamma$ is a flat fiber bundle over $G / \Gamma$ with fiber $M$, i.e. locally a product of $G / \Gamma$ with $M$. Let $h_{M}, h_{G / \Gamma}$ and $h_{(G \times M) / \Gamma}$ be the entropy functions for the actions of $\Gamma$ on $M$ and $G$ on $G / \Gamma$ and $(G \times M) / \Gamma$ respectively. Note that since $h_{M}$ is given as in Theorem 2.2, $h_{M}$ is defined for all $g \in G$, even though we only have an action of $\Gamma$. By showing that the superrigidity representation preserves the direct sum decomposition of $T((G \times M) / \Gamma)$, we prove the following formula:

Theorem 2.3. $h_{(G \times M) / \Gamma}(\gamma)=h_{G / \Gamma}(\gamma)+h_{M}(\gamma)$

Proof. Since the $G$ action preserves the splitting $T((G \times M) / \Gamma)=T(\mathfrak{G}) \times T M$, we can view the derivative cocycle as taking values in the bundle $P(\mathfrak{G}) \times P(M)$ of frames respecting this decomposition. The cocycle into $P(\mathfrak{G})$ is given by the adjoint action of $G$, exactly as in the case of $G$ acting on $G / \Gamma$. We are therefore reduced to studying the $G$ action on the bundle $(G \times P(M)) / \Gamma \rightarrow(G \times M) / \Gamma$.

Applying superrigidity for cocycles as described above, we see that possibly after passing to a finite ergodic extension of $(G \times M) / \Gamma$, there is a measurable section $s:(G \times M) / \Gamma \rightarrow(G \times P(M)) / \Gamma$ and a linear representation $\pi: G \rightarrow S L_{n} \mathbb{R}$, where $n=\operatorname{dim} M$ such that

$$
s(g x)=g s(x) \pi(g)^{-1} c(x, g)
$$

for all $g \in G$ and almost every $x \in(G \times M) / \Gamma$, where $c$ is a cocycle taking values in a compact group which commutes with $\pi(G)$. Note that $\pi$ being trivial is equivalent to the algebraic hull of the cocycle being compact. This shows us that to compute the entropy of the $G$ action on $(G \times M) / \Gamma$ we can take $\sigma=A d \oplus \pi$ and compute as in Theorem 2.1. To complete the proof, we need only see that $\pi$ can also be used via Theorem 2.2, to compute the entropy of the $\Gamma$ action on $M$. 
Let $[g] \in G / \Gamma$, then $g \Gamma g^{-1}$ acts on the $M$ fiber over $[g]$ in $(G \times M) / \Gamma$ via translation to the fiber over the identity, the given $\Gamma$ action on $M$, and then translation back. Since $s$ is defined for almost all $x \in(G \times M) / \Gamma$, writing $x=[g, m]$ and applying Fubini's theorem, we see that for almost all $\left[g_{0}\right] \in G / \Gamma, s$ is defined for almost every $\left[g_{0}, m\right]$ in the $M$ fiber over $\left[g_{0}\right]$. Possibly after conjugating the action by $g_{0}^{-1}$, we can therefore assume that $s$ is defined for almost every $m \in M$ in the fiber over $[e]$. Restricting $s$ to $[e, m]$ and looking only at $\gamma \in \Gamma$, the formula above implies that

$$
\left.s(\gamma[e, m])=\gamma s([e, m]) \pi^{-1}(\gamma) c([e, m]), \gamma\right)
$$

for all $\gamma \in \Gamma$ and almost every $m \in M$. This shows that if we use the restriction of $s$ to trivialize $P(M) \rightarrow M$ we can compute the Lyapunov exponents of the derivative cocycle via those of $\pi^{-1}(\gamma) c(m, \gamma)$ and since the image of $c$ is compact, we can compute the entropy of the $\Gamma$ action on $M$ via the eigenvalues of the representation $\pi$.

Remark. We have actually proved more than just the formula. We have shown that, for the derivative cocycle, the superrigidity representation for the induced action is cohomologous to the direct sum of the superrigidity representation for the $\Gamma$ action and the representation $A d_{G}$.

Corollary 2.4. Suppose $\Gamma$ acts on a compact manifold $M$ preserving a unimodular rigid geometric structure. Assume further that the action is ergodic and that $h_{(G \times M) / \Gamma}(g)=h_{G / \Gamma}(g)$, for all $g \in G$. Then the action is Riemannian isometric.

Proof. By Theorem 2.3, this implies that $h_{M}(\gamma)=0$ for all $\gamma \in \Gamma$. Either by looking at the proof of that theorem or via Theorem 2.2, this implies the algebraic hull of the derivative cocycle is compact. By the proof of Theorem 4.5 from [Z4] this implies that there is a $\Gamma$ invariant finite measure on $P^{k}(M)$, the $k^{\text {th }}$ order frame bundle, for any $k$. In fact this measure is the volume on a (measurable) $K$ bundle over $M$, where $K$ is a compact subgroup of the higher order frame group. However, Gromov has observed that any closed subgroup of the isometry group of a rigid geometric structure is proper and free on $P^{k}(M)$ for $k$ large enough [G, Section 0.4]. Since the closure of $\Gamma$ acts properly on a finite measure space, the closure of $\Gamma$ in the automorphism group of the structure is compact (see [Z4] proof of Theorem 5.2 for more details) and therefore the action is isometric.

\section{Products of geometric structures}

In this section we will develop the ideas we need concerning geometric structures, particularly products of structures on (local) products of manifolds. Throughout $D_{n}^{s}$ will denote the group of $s$-jets of diffeomorphisms of $\mathbb{R}^{n}$ fixing 0 and $P^{s}(M)$ 
will be the $s^{\text {th }}$ order frame bundle of the manifold $M$. This is a $D_{n}^{s}$ principal bundle where $n=\operatorname{dim} M$.

Definition 3.1. A geometric structure, $\omega$, on a manifold $M$, with $\operatorname{dim} M=n$, consists of:

a) an algebraic variety $V$ on which $D_{n}^{s}$ acts for some $s$

b) a map $\omega: P^{s}(M) \rightarrow V$ which is $D_{n}^{s}$ equivariant.

The structure is said to be of order $s$ and is $C^{r}\left(C^{\omega}\right)$ when the map $w$ is $C^{r}\left(C^{\omega}\right)$. The structure is called unimodular if it defines a volume form on $M$.

If $V=D_{n}^{s} / H$ where $H<D_{n}^{s}$ is an algebraic subgroup, this reduces to the notion of a $H$ structure. Note that Gromov calls $\omega$ as above a rigid $A$-structure and defines a more general notion of rigid geometric structure.

Given $\omega$, let $\operatorname{Iso}_{\omega}^{r}(x)$ be the set of $r$-jets of diffeomorphisms of $M$ fixing the point $x$, and fixing $\omega$ up to order $r$ at $x$.

Definition 3.2. A geometric structure is called rigid of order $r+1$ if the natural map $\operatorname{Iso}^{r+1}(x) \rightarrow \operatorname{Iso}^{r}(x)$ is injective, for all $x \in M$. If we are not concerned with the order, we simply call the structure rigid.

Example 3.3. a) A pseudo-Riemannian metric is rigid of order 1 , since it determines a total framing of $P^{1}(M)$.

b) Let $G$ be a simple Lie group, $\Gamma<G$ a lattice. Since the $G$ action on $G / \Gamma$ preserves the Killing form on $T(G / \Gamma)$, it preserves a rigid geometric structure: the pseudo-Riemannian metric defined by the Killing form.

Let $V$ be a smooth manifold of dimension $n$ and let $P_{n}^{s} V$ be the space of $s$-jets of diffeomorphisms of $\left(\mathbb{R}^{n}, 0\right)$ into $V$. If $D_{n}^{r}$ acts on $V$ then $D_{n}^{r+s}$ acts on $P_{n}^{s} V$. Furthermore if $V$ is an algebraic variety, then so is $P_{n}^{s} V$ and the $D_{n}^{r+s}$ action is algebraic if the $D_{n}^{r}$ action is. Given a geometric structure $\omega: P^{r}(M) \rightarrow V$, we have a naturally defined map $\omega^{s}: P^{r+s}(M) \rightarrow P_{n}^{s}(V)$ which we call the $s^{t h}$ prolongation of the geometric structure. It is easy to verify that $\omega^{s}$ is a geometric structure and if $\omega$ is rigid so is $\omega^{s}$. (See $[\mathrm{Fe}]$ or $[\mathrm{G}]$ for details).

Let $M$ and $N$ be differentiable manifolds of dimension $m$ and $n$ respectively. Suppose $a: P^{r_{1}}(M) \rightarrow V_{1}$ and $b: P^{r_{2}}(N) \rightarrow V_{2}$ are geometric structures. By passing to a prolongation of the structure of lower order, we may assume $r_{1}=r_{2}$. To produce a geometric structure on $M \times N$, we begin with the map

$$
a \times b: P^{r}(M) \times P^{r}(N) \rightarrow V_{1} \times V_{2}
$$

and, using the inclusion $D_{m}^{r} \times D_{n}^{r}<D_{m+n}^{r}$, induce this to a map $(a \times b)^{\prime}:\left(D_{n+m}^{r} \times P^{r}(M) \times P^{r}(N)\right) /\left(D_{n}^{r} \times D_{m}^{r}\right) \rightarrow\left(D_{n+m}^{r} \times V_{1} \times V_{2}\right) /\left(D_{n}^{r} \times D_{m}^{r}\right)$. Given an action of a closed subgroup $H<L$ on a space $X$, we can define the induced action of $L$. The space acted upon is $(L \times X) / H$ where the $H$ action we 
divide by is given by $(l, x) h=\left(l h, h^{-1} x\right)$. The $L$ action on the space is defined by the left $L$ action on the first factor, which is well-defined on the quotient since it commutes with the $H$ action defined above. Note that this definition only works for left actions of $H$ on $X$, analogous definitions allow us to induce right actions to right actions. If the action of $H$ on $X$ is algebraic and $H$ is an algebraic subgroup of an algebraic group $L$, then the induced action is an algebraic action on an algebraic variety. Also note that $\left(D_{n+m}^{r} \times P^{r}(M) \times P^{r}(N)\right) /\left(D_{n}^{r} \times D_{m}^{r}\right)$ with the induced action is canonically isomorphic to $P^{r}(M \times N)$. Therefore $(a \times b)^{\prime}$ is in fact a map

$$
(a \times b)^{\prime}: P^{r}(M \times N) \rightarrow V
$$

where $V=\left(D_{n+m}^{r} \times V_{1} \times V_{2}\right) /\left(D_{n}^{r} \times D_{m}^{r}\right)$ is an algebraic variety with an algebraic action of $D_{n+m}^{r}$ for which $(a \times b)^{\prime}$ is equivariant.

Proposition 3.4. If $a: P^{r}(M) \rightarrow V_{1}$ and $b: P^{s}(M) \rightarrow V_{2}$ are rigid geometric structures, then the product structure $(a \times b)^{\prime}: P^{\max (r, s)}(M \times N) \rightarrow V$ is also rigid.

Proof. By passing to a prolongation of one structure, it suffices to consider $r=$ $s$. A $k$-jet of a diffeomorphism fixing a point $x$ leaves $(a \times b)^{\prime}$ invariant if and only if it leaves $(a \times b)^{\prime} \mid P^{r}(M) \times P^{r}(N)$ invariant. This restriction is exactly $a \times b: P^{r}(M) \times P^{r}(N) \rightarrow V_{1} \times V_{2}$. Direct computation of derivatives shows that $\mathrm{Iso}_{a \times b}^{r}=\mathrm{Iso}_{a}^{r} \times \mathrm{Iso}_{b}^{r}$ where we view an $r$-jet, $j^{r}(f)$ (resp. $\left.j^{r}(g)\right)$ of a diffeomorphism of $N$ at $x_{2}$ (resp. $M$ at $\left.x_{1}\right)$ as an $r$-jet of $M \times N$ at $\left(x_{1}, x_{2}\right)=x$ as $j^{r}(\operatorname{Id} \times f)$ (resp. $\left.j^{r}(g \times \mathrm{Id})\right)$.

If a structure $\omega$ is rigid of order $r$, it follows that $\operatorname{Iso}_{\omega}^{r+j} \rightarrow \operatorname{Iso}_{\omega}^{r}$ is injective for all $j \geq 1$ (see [G, remark on page 68] or [Fe, Proposition 3.1]). Therefore, it follows that $(a \times b)^{\prime}$ is rigid (and that it is rigid of the same order as whichever of $a$ and $b$ has the higher order of rigidity).

Corollary 3.5. Let $G$ be a semisimple Lie group, $\Gamma<G$ a lattice. Suppose $\Gamma$ acts on $M$ preserving a rigid geometric structure $\psi$. Then the $G$ action on $(G \times M) / \Gamma$ also preserves a rigid geometric structure.

Proof. The pseudo-Riemannian metric defined by the Killing form on $G$ is biinvariant and so defines a $G \times G$ invariant structure $a: P^{1}(G) \rightarrow V$. By the proposition above, $(a \times \psi)^{\prime}$ is a rigid geometric structure on $G \times M$ which is invariant under the right and left $G$ actions and the $\Gamma$ action on $M$. Therefore it descends to a $G$ invariant geometric structure $\varphi$ on $(G \times M) / \Gamma$. Since $(a \times \psi)^{\prime}$ is rigid so is $\varphi$, since rigidity is a local property. 


\section{Proofs}

The proof of Theorem 1.1 now follows from Corollary 2.4 and an estimate of the "entropy" of Gromov's representation computed in [Z5]. Given any finite dimensional $G$ module $D$, we can define the entropy of the $G$ action by $h_{D}(g)=$ $\Sigma \log ^{+}\|\lambda\|$ where the sum is taken over eigenvalues for the action of $g$ on $D$.

Assume $G$ is a simple non-compact Lie group and $G$ acts on a compact manifold $X$ preserving a volume form and a rigid geometric structure $\omega$. Let $\tilde{G}$ be the universal cover of $G$. The action on $X$ lifts to a $\tilde{G}$ action on $\tilde{X}$, the universal cover of $X$. Let $\tilde{\omega}$ be the lift to $\tilde{X}$ of the geometric structure. Then $\tilde{G}$ clearly preserves $\tilde{\omega}$. In [G] Gromov constructs a linear representation $\sigma$ of $\pi_{1}(X)$ on the set of Killing fields $V$ of $\tilde{\omega}$ centralizing the $\tilde{G}$ action i.e. a representation $\sigma: \pi_{1}(X) \rightarrow \operatorname{Aut}(V)$. Let $H$ be the Zariski closure of $\sigma\left(\pi_{1}(X)\right)$. Gromov proves that $H$ contains a group $G^{\prime}$ locally isomorphic to $G$, making $\mathfrak{h}=\operatorname{Lie}(H)$ into a $G^{\prime}$ module via the restriction of $\operatorname{Ad}_{H}$ to $G^{\prime}$. For an accessible presentation of the proof see [Z3]. In this context, Lemma 5.1 of $[\mathrm{Z} 5]$ shows that:

$$
h_{\mathfrak{h}}(g) \geq h_{X}(g) \quad \forall g \in G .
$$

Proof of Theorem 1.1. As discussed in the introduction, we apply Gromov's result discussed above to the $G$ action on $(G \times M) / \Gamma$, assuming $G$ is simply connected. The rigid structure, $\omega$, on $(G \times M) / \Gamma$ is as described in Corollary 3.5. Let $\Lambda=$ $\pi_{1}((G \times M) / \Gamma)$ and recall that

$$
1 \rightarrow \pi_{1}(M) \rightarrow \Lambda \stackrel{p}{\longrightarrow} \Gamma \rightarrow 1 .
$$

By Proposition 6.1 of [F], $\Lambda$ is isomorphic to the group of lifts of the $\Gamma$ action on $M$ to $\tilde{M}$ and the action on $G \times \tilde{M}$ is given by $(g, m) \lambda=\left(g p(\lambda)^{-1}, \lambda m\right)$. We will also need one obvious fact about the lift of our rigid structure $\omega$ to $G \times \tilde{M}$. Since here it is $G$ bi-invariant by construction, the Lie algebra of vector fields, $\mathfrak{g}$, generating the right $G$ action on $G$ (i.e. the left invariant vector fields) are Killing fields of $\tilde{\omega}$ that centralize the lift of our $G$ action from $(G \times M) / \Gamma$. These vector fields are clearly invariant under the $\pi_{1}(M)$ action on $G \times \tilde{M}$. In Gromov's construction of $\sigma$, the group $G^{\prime}<H$ is described quite explicitly. It's Lie algebra $\mathfrak{g}^{\prime}$ is a Lie algebra of Killing fields of $\tilde{\omega}$ commuting with the action of $G$. Even more is true. Let $\mathfrak{n}$ be the algebra of Killing fields of $\tilde{\omega}$ normalizing the $G$ action. Then $\mathfrak{n}=\mathfrak{g} \oplus V$ where $V$ is the algebra of Killing fields commuting with the $G$ action, and $\mathfrak{g}$ is the algebra of Killing fields which are derivatives of the $G$ action. For a point $x_{0} \in X$, Gromov proves that there exists a Lie algebra $\mathfrak{g}_{\Delta}<\mathfrak{n}$ such that 1) elements of $\mathfrak{g}_{\Delta}$ fix $x_{0}$ and 2) $\mathfrak{g}_{\Delta}$ is isomorphic to $\mathfrak{g}$ and is, in fact, the graph of a isomorphism $\tau: \mathfrak{g} \rightarrow V$. Then $\mathfrak{g}^{\prime}$ is the image $\tau(\mathfrak{g})<V$. Conditions 1) and 2) above canonically define $\mathfrak{g}_{\Delta}$ and $\mathfrak{g}^{\prime}$. In our situation, we can realize $\mathfrak{g}_{\Delta}$ as those Killing fields at $x_{0}=\left(\left[g_{0}\right], m\right)$ 
generated by the action of $g_{0} \circ \mathrm{Ad} G \circ g_{0}^{-1}$ and $\mathfrak{g}^{\prime}$ as the vector fields generating the right $G$ action on $G \times M$.

The representation $\sigma$ of $\Lambda$ is defined on all Killing fields of $\tilde{\omega}$ commuting with the left $G$ action, and so contains as a subrepresentation the representation $\sigma^{\prime}$ on the Lie algebra $\mathfrak{g}^{\prime}$ of left invariant vector fields on $G$. Since $\pi_{1}(M)$ acts trivially on these vector fields, it is easy to see that this representation is just $\left.\left(\operatorname{Ad}_{G}\right)\right|_{\Gamma} \circ p$. In particular, the Zariski closure of $\sigma^{\prime}(\Lambda)$ is a group locally isomorphic to $G$.

Let $H$ be the Zariski closure of $\sigma(\Lambda)$ and $L \triangleleft H$ be the Zariski closure of $\sigma\left(\pi_{1}(M)\right)$. Recall that $H$ and $L$ are subgroups of $\operatorname{Aut}(V)$ where $V$ is the Lie algebra of Killing fields of $\omega$ commuting with the $G$ action on $G \times \tilde{M}$.

Let $\mathfrak{h}=\operatorname{Lie}(H), \ell=\operatorname{Lie}(L)$. Then $\ell<\mathfrak{h}$ is an ideal and is also a $G^{\prime}$ submodule for the $G^{\prime}$ action on $\mathfrak{h}$ given by $\left.\left(\mathrm{Ad}_{H}\right)\right|_{G^{\prime}}$. To prove Theorem 1.1, it suffices to see that if $\ell$ is a trivial $G$ module, then the $\Gamma$ action is isometric.

If $\ell$ is a trivial $G^{\prime}$ module, then $h_{\mathfrak{h}}(g)=h_{\mathfrak{h} / \ell}(g) \forall g \in G^{\prime}$. Let $\pi: H \rightarrow H / L$ be the natural projection. Then $\pi \circ \sigma$ is a homomorphism of $\Gamma$ into an algebraic group, which has Zariski dense image, since $H$ is the Zariski closure of $\sigma(\Lambda)$. Now $\pi \circ \sigma$ contains $\pi \circ \sigma^{\prime}$ as a subhomomorphism. Clearly $\pi_{1}(M)$ acts trivially on the subspace $\mathfrak{g}^{\prime}$ of Killing fields on which $\sigma^{\prime}$ is defined, so $L$ acts trivially there as well. (Acting trivially is an algebraic condition.) This means that $\sigma^{\prime}(\Lambda) \cap L$ is trivial, so the Zariski closure of $\pi \circ \sigma^{\prime}(\Lambda)$ is also a group locally isomorphic to $G$.

Now $\pi \circ \sigma$ factors through a homomorphism of $\Gamma$, a lattice in simple Lie group with $\mathbb{R}-\operatorname{rank}(G) \geq 2$, so the Zariski closure of $\pi \circ \sigma(\Lambda)$ is, by Margulis' Superrigidity Theorem, locally isomorphic to a product of $G$ with a compact semi-simple group. Therefore it is a compact extension of the Zariski closure of $\pi \circ \sigma^{\prime}(\Lambda)$. So $\mathfrak{h} / \ell$ as a $G^{\prime}$-module is just $\mathfrak{g} \oplus \mathfrak{k}$, where $\mathfrak{k}$ is a compact semi-simple Lie algebra that is necessarily trivial as a $G^{\prime}$ module. Therefore $h_{\mathfrak{h} / \ell}(g)=h_{\mathfrak{g} \oplus \mathfrak{k}}(g)=h_{\mathfrak{g}}(g)$, and furthermore $h_{\mathfrak{h}}(g)=h_{\mathfrak{g}}(g), \forall g \in G$. But by $(*)$ above and Theorem 2.3

$$
h_{\mathfrak{h}}(g) \geq h_{(G \times M) / \Gamma}(g)=h_{G / \Gamma}(g)+h_{M}(g)=h_{\mathfrak{g}}(g)+h_{M}(g)
$$

for all $g \in G$. Since $h_{\mathfrak{h}}(g)=h_{\mathfrak{g}}(g), \forall g \in G$, we see that $h_{(G \times M) / \Gamma}(g)=h_{G / \Gamma}(g)$, $\forall g \in G$, so by Corollary 2.4 the $\Gamma$ action on $M$ is isometric.

In the equation above, we refer to $h_{M}(g)$ where only $\Gamma$, and not $G$, acts on $M$. However, the equation still makes sense, since the entropy of the $\Gamma$ action on $M$ is really computed, as described in Theorem 2.2 via the eigenvalues of a linear representation of $G$. So by $h_{M}(g)$ here, we really mean the entropy of this linear representation, or 0 , if the representation is trivial. The equation is formally justified by the remark following Theorem 2.3 .

In $[\mathrm{Z} 5]$, the second author proves:

Theorem 4.1. Let $X$ be a compact real analytic manifold with a real analytic unimodular rigid geometric structure. Let $G$ be a connected simple Lie group with 
$\mathbb{R}-\operatorname{rank}(G) \geq 2$ and suppose $G$ acts analytically and ergodically on $X$ preserving the structure. Further assume the action is engaging. Then there is a finite ergodic extension $X^{\prime}$ of $X$ and a measurable $G$ equivariant $\operatorname{map} \varphi: X^{\prime} \rightarrow K \backslash H / \Lambda$ where $G<Z_{K}(H)$ and $K$ is compact. Furthermore $h_{X}(g)=h_{K \backslash H / \Lambda}(g)$ for all $g \in G$.

The quotient $\varphi: X^{\prime} \rightarrow K \backslash H / \Lambda$ is constructed using the techniques of [LZ] from Gromov's representation of $\pi_{1}(X)$. Our Theorem 1.3 follows by combining the theorem above, the entropy results for induced actions of Section 2, and results of $[\mathrm{F}]$ on constructing quotients of lattice actions and induced actions.

First, we recall some of the ideas used to prove Theorem 4.1. Let $\sigma: \pi_{1}(X) \rightarrow$ $G L_{n}(\mathbb{R})$ be Gromov's representation discussed above. Let $A$ be the finitely generated $\mathbb{Q}$ algebra such that $\sigma(\gamma) \in G L(n, A)$ for all $\gamma \in \pi_{1}(X)$. Let $\overline{\mathbb{Q}}$ denote the algebraic integers. In [LZ], a specialization $\psi: A \rightarrow \overline{\mathbb{Q}}$ is constructed such that the map induced by $\psi$ on $G L_{n}(A)$ is an isomorphism when restricted to $\sigma\left(\pi_{1}(X)\right)$ and $\psi \circ \sigma\left(\pi_{1}(X)\right)$ is an $\mathfrak{s}$-arithmetic subgroup of a perfect $\mathbb{Q}$-group $H$. Furthermore $\psi \circ \sigma\left(\pi_{1}(X)\right) \cap H_{\mathbb{Z}}=\Lambda$ is of finite index in $H_{\mathbb{Z}}$ and is a lattice in $H$. Lubotzky and Zimmer also show that there is a finite ergodic extension $X^{\prime}$ of $X$ such that there is a measurable map $\varphi: X^{\prime} \rightarrow K \backslash H / \Lambda$. The key idea of [Z5] is to use the way in which $\sigma$ and $\psi$ are constructed to see that the entropies are equal.

Proof of Theorem 1.3. We apply Theorem 4.1 to the action of $G$ on $(G \times M) / \Gamma$. We can do this despite the fact that $(G \times M) / \Gamma$ is not compact, since this is only used to show that certain cocycles are quasi-integrable. (See the discussion following Theorem 2.2 for entropy considerations and the derivative cocycle and Section 7 of $[\mathrm{F}]$ for cocycles coming from representations of the fundamental group.) Letting $\hat{\sigma}=\psi \circ \sigma$, as discussed above, the proof of the main theorem of [LZ] implies that $\hat{\sigma}\left(\pi_{1}((G \times M) / \Gamma)\right)$ is $\mathfrak{s}$-arithmetic in an algebraic $\mathbb{Q}$-group $H$, and if $\Lambda=$ $\hat{\sigma}\left(\pi_{1}((G \times M) / \Gamma)\right) \cap H_{\mathbb{Z}}$, that we have a map from a finite extension $X$ of $(G \times M) / \Gamma$ to $K \backslash H / H_{\mathbb{Z}}$ that is $G$ equivariant.

As in the proof of Theorem 1.1, let $L^{\prime}$ be the Zariski closure of $\sigma\left(\pi_{1}(M)\right)$ and let $H^{\prime}$ be the Zariski closure of $\sigma\left(\pi_{1}((G \times M) / \Gamma)\right)$. As above let $\sigma^{\prime}$ be the subrepresentation of $\sigma$ defined on the vector fields $\mathfrak{g}$ given by differentiating the right action of $G$ on $G \times \tilde{M}$. Recall that $\sigma^{\prime}\left(\pi_{1}((G \times M) / \Gamma)\right) \cap L^{\prime}=\emptyset$, and since $\sigma^{\prime}\left(\pi_{1}((G \times M) / \Gamma)\right)$ is virtually isomorphic to $\Gamma$, it follows that $\left[\sigma\left(\pi_{1}(M)\right): \sigma\left(\pi_{1}((G \times M) / \Gamma)\right)\right]=\infty$, unless $\sigma\left(\pi_{1}(M)\right)$ is finite and the $\Gamma$ action on $M$ is isometric. Since the specialization $\psi$ is an isomorphism on $\sigma\left(\pi_{1}((G \times M) / \Gamma)\right)$, it is immediate that $\left[\hat{\sigma}\left(\pi_{1}(M)\right)\right.$ : $\left.\hat{\sigma}\left(\pi_{1}((G \times M) / \Gamma)\right)\right]=\infty$ as well. It is clear from the discussion above of the proof of Theorem 4.1 that $H$ is the Zariski closure of $\hat{\sigma}\left(\pi_{1}((G \times M) / \Gamma)\right)$. Let $L \triangleleft H$ be the Zariski closure of $\hat{\sigma}\left(\pi_{1}(M)\right)$.

By Theorem 8.1 of [F], we see that $H=(G \times C) \ltimes L$ and $\sigma\left(\pi_{1}((G \times M) / \Gamma)\right)=$ $\Gamma \ltimes \Delta$ (up to finite index) where $\Delta=L \cap \sigma\left(\pi_{1}((G \times M) / \Gamma)\right.$ and $C$ is compact. We have a measurable map $\varphi: X \rightarrow K \backslash H / \Lambda$ and $X$, as a finite extension of $(G \times M) / \Gamma$, 
is $\left(G \times M^{\prime}\right) / \Gamma^{\prime}$ where $M^{\prime}$ is finite (possibly disconnected) cover of $M$ and $\Gamma^{\prime}<\Gamma$ is of finite index. (See [F], proof of Lemma 4.3, for details.) Let $\Delta_{\infty}=\Delta \cap L_{\mathbb{Z}}$. Then by Theorem 8.5 of [F], we see that $K \backslash H / \Lambda=K \backslash(G \times C) \ltimes L / \Gamma^{\prime} \ltimes \Delta_{\infty}$ and that $\varphi$ takes almost every $M^{\prime}$ fiber of $X$ to a $K \backslash C \times L / \Delta_{\infty}$ fiber in $K \backslash H / \Lambda$.

The $G$ action on $K \backslash(G \times C) \ltimes L / \Gamma^{\prime} \ltimes \Delta_{\infty}$ is isomorphic to the $G$ action induced from the $\Gamma^{\prime}$ action on $K \backslash C \times L / \Delta_{\infty}$. So by Theorem 2.3 above, the entropy function for the $\Gamma^{\prime}$ action on $M^{\prime}$ and for the $\Gamma^{\prime}$ action on $K \backslash C \times L / \Delta_{\infty}$ must be equal, since by Theorem 4.1, the entropy functions for the induced actions are equal.

\section{References}

[F] D. Fisher, On the arithmetic structure of lattice actions on compact spaces, to appear Ergodic Th. and Dyn. Sys.

[Fe] R. Feres, Rigid geometric structures and actions of semi-simple Lie groups, preprint, to appear in Proceedings of Strasbourg Conference, (ed. P. Foulon).

[G] M. Gromov, Rigid transformation groups, Géometrie Différentiale (D. Bernard and Y. Choquet-Bruhat, eds.), Hermann, Paris 1988.

[L] J. Lewis, The algebraic hull of a measurable cocycle, to appear Geom. Ded.

[LZ] A. Lubotzky and R. J. Zimmer, Arithmetic structure of fundamental groups and actions of semisimple groups, Topology 40 (2001), 851-869.

[P1] Y. Pesin, Lyapunov characteristic exponents and smooth ergodic theory, Russian Math. Surveys 32 (1977), 55-114.

[P2] Y. Pesin, personal communication.

[Z1] R. J. Zimmer, Ergodic Theory and Semisimple Groups, Birkhäuser, Boston, 1984.

[Z2] R. J. Zimmer, Superrigidity, Ratner's theorem, and fundamental groups, Israel J. Math. 74 (1991), 199-207.

[Z3] R. J. Zimmer, Automorphism groups and fundamental groups of geometric manifolds, Proc. Symp. in Pure Math. 54 (1993), vol. 3.

[Z4] R. J. Zimmer, Lattices in semisimple groups and invariant geometric structures of compact manifolds, Discrete Groups in Geometry and Analysis (ed. Roger Howe), Birkhäuser, Boston, 1987.

[Z5] R. J. Zimmer, Entropy and arithmetic quotients for simple automorphism groups of geometric manifolds, to appear Geom. Ded.

[Z6] R. J. Zimmer, On the algebraic hull of an automorphism group of a principal bundle, Comm. Math. Helv. 65 (1990), 375-387.

David Fisher

Department of Mathematics

Yale University

PO Box 208283

New Haven, CT 06520-8283

USA

e-mail: david.fisher@yale.edu
Robert J. Zimmer

Department of Mathematics

University of Chicago

Chicago

IL 60637

USA

(Received: December 14, 2000) 